1 Past and future perspectives on mathematical models of tick-borne pathogens.

2 Norman, R.A. ${ }^{1}$, Worton, A.J. ${ }^{2}$ and Gilbert, L. ${ }^{3}$

3

4

5

6

7

8 Running title: Models of tick-borne pathogens.

9 Corresponding author: Rachel Norman, School of Natural Sciences, University of Stirling,

10 Stirling FK9 4LA. Telephone: 01786 467466. Fax: 01786 464551. Email:

11 r.a.norman@stir.ac.uk 
14 Ticks are vectors of pathogens which are important both with respect to human health and 15 economically.

16 They have a complex lifecycle requiring several blood meals throughout their life. These

17 blood meals take place on different individual hosts and potentially on different host species.

18 Their lifecycle is also dependent on environmental conditions such as the temperature and 19 habitat type.

20 Mathematical models have been used for the more than 30 years to help us understand how

21 tick dynamics are dependent on these environmental factors and host availability.

22 In this paper we review models of tick dynamics and summarise the main results. This summary is split into two parts, one which looks at tick dynamics and one which looks at tick

24 borne-pathogens.

25 In general, the models of tick dynamics are used to determine when the peak in tick densities

26 is likely to occur in the year and how that changes with environmental conditions. The

27 models of tick borne pathogens focus more on the conditions under which the pathogen can persist and how host population densities might be manipulated to control these pathogens.

29 In the final section of the paper we identify gaps in the current knowledge and future modelling approaches.

32 Keywords:

33 Tick-borne pathogen, mathematical model, Louping ill, Lyme disease. 
35 Ticks are the most important vectors of zoonotic disease-causing pathogens in Europe, 36 transmitting the tick-borne encephalitis (TBE) complex of viruses, Anaplasma 37 phagocytophyllum, Babesia and Rickettsia species and Borrelia burgdorferi sensu lato, the 38 complex of bacteria that cause Lyme borreliosis, amongst others. Ixodes ricinus L. ticks are 39 particularly implicated in pathogen transmission because they are almost ubiquitous across

40 Europe and are generalist feeders, which allows for pathogen transmission among different

41 host species. I. ricinus are increasing in number and range in many parts of northern Europe

42 (reviewed by Medlock et al. 2013).

43 In any given geographical region tick population dynamics are dependent on a number of 44 biotic and abiotic factors including the density of different host species, and other factors that 45 influence survival and activity such as temperature and humidity and vegetation types, the 46 latter of which provide habitats for different hosts and create different microclimates.

47 Mathematical models have been used extensively to predict the dynamics of tick populations under different conditions including climate change. However, high tick densities do not necessarily mean high prevalence or risk of tick-borne pathogens, since this is dependent not only ticks but also competent transmission hosts. Therefore, models have also been used to

51 predict the tick-borne pathogen dynamics and the theoretical effectiveness of different tick52 borne pathogen control methods under different environmental or management scenarios. In this paper we will review the use of those models for different systems, summarise they key results in different contexts and discuss possible future directions of mathematical modelling of tick-borne pathogens. 


\section{MATHEMATICAL MODELS OF TICK POPULATION DYNAMICS}

58 Although there are a number of different tick species globally this review will focus on $I$. ricinus and we will specify when we cite any papers which refer to other species.

The I. ricinus life cycle develops from the egg, through two immature stages (larvae and nymph) to the adult stage. Each immature stage requires a blood meal from a suitable vertebrate host before developing to the next stage and the adult female requires a blood meal before producing eggs. Adult females feed primarily on large mammals such as deer, sheep or hares whilst the immature stages can also feed on smaller vertebrates such as mice, voles and birds (e.g. Gray 1998). The I. ricinus life cycle usually takes 3-4 year to complete (Fig. 1).

In winter ticks often enter behavioural diapause induced by cold and/or short day length (Randolph et al. 2002; but see Gray 1987). Therefore, tick activity is highly seasonal with ticks in northern Europe being active mainly between spring and autumn when temperatures are warm enough. Activity is inhibited by cold temperatures but increases with temperature up to a limit $\left(12-20^{\circ} \mathrm{C}\right.$ depending on population e.g. Gilbert et al. (2014); Tomkins et al. (2014)). Tick host-seeking (questing) activity can also be inhibited by low relative humidity or high saturation deficits (this is a function of relative humidity and temperature and gives an estimate of the drying power of the air; Perret et al. 2000). After feeding, ticks also become inactive due to physiological diapause while they develop into the next stage (Randolph et al. 2002).

One of the first mathematical models developed to describe tick population dynamics was published in 1981 (Gardiner, et al. 1981). This study used empirical data from experiments to predict how tick development times depend on temperature. They did not put this into a 
formal predictive modelling framework but they did try to determine functional relationships between development time and different measures of temperature (i.e. air and soil temperature). In particular they looked at how experimentally predicted development times estimated in the laboratory translated to the field where temperature fluctuations are much less predictable. They found that soil temperatures recorded at a depth of 50mm are useful predictors for larval and nymphal development phases. In terms of egg development time they found that air temperatures are useful for predicting the development time of eggs laid in the spring but soil temperature is a better predictor for those laid in autumn. They suggested that this might be because during diapause eggs may be conditioned to develop according to the temperature of their environment rather than air temperature.

Mount and Haile (1989) developed a computer simulation model of the American dog tick Dermacentor variabilis (Say). This model simulated the effects of environmental variables such as ambient temperature, habitat and host density on American dog tick population dynamics. They validated the model by comparing its predictions with empirical data from Virginia, Maryland and Massachusetts. The authors concluded that the model produced acceptable values for equilibrium population densities and seasonal activity patterns and went on to extend this model to include Rocky Mountain spotted fever dynamics (Cooksey et al. 1990).

Over the last 40 years Sarah Randolph and collaborators have written a large number of papers on tick biology and population dynamics. These are largely empirical; however there are also some which model tick population dynamics. The first of these came in 1997 (Randolph and Rogers 1997) where they presented a simulation model of the African tick Rhipicephalus appendiculatus. This simulation model incorporated temperature dependent rates of egg production and development, climate driven density independent mortality rates 
and density dependent regulation of both nymphs and adults. The model successfully described both the seasonality and annual range of variation in numbers of each tick stage observed at each of four test sites in Uganda, Burundi and South Africa.

In 2002 Randolph et al. used empirical data on tick counts, various microclimatic factors and fat contents of ticks to create a population model explaining seasonality of I. ricinus in the UK. This study showed large variation in questing activity between years, but the date of questing (i.e. host-seeking activity) in one year was used to predict the start of questing for the next stage the following year, with reasonable accuracy. This was an important paper that also found evidence of two cohorts of ticks within a life stage within a season. Those nymphs with higher relative fat contents had emerged and become active more recently than those with lower fat contents. The suggestion was that spring-questing nymphs had overwintered, having fed as larvae the previous late summer or autumn; meanwhile autumn-questing nymphs had fed as larvae in the spring of the same calendar year.

More recently, Dobson et al. (2011) used a stage-classified Leslie matrix model to break the tick life cycle into the key parts, with a particular focus on two types of diapause: developmental and behavioural, with the latter being important in determining how many times a year an individual tick might feed. This model was then used by Dobson and Randolph (2011) to make long-term predictions of the effects of host densities, climate and acaricide treatment of hosts on tick populations.

In 2005 Ogden et. al. developed a model of Ixodes Scapularis Say (1821) in which tick development rates were modelled as temperature dependent time delays. Time spent in egg and engorged tick states and questing activities were all temperature dependent. The parameters were estimated using data taken from Ogden et.al. (2004). The model was validated using data from Ontario and Maryland and in both cases the observed seasonal 
activity patterns were predicted by the model. The models were then used to predict theoretical geographical limits for the establishment of I. Scapularis in Canada. The model predicted that the temperature conditions which are suitable for the tick are wider than the existing distribution, implying that there is potential for spread.

At a similar time a different group used an age-structured stochastic model to describe the dynamics of tick populations (Hancock et al. 2011). They focused on the effect of temperature on the development between each stage of the tick life cycle, i.e. from egg to larva, larva to nymph, nymph to adult, and adult laying eggs. This model also introduced pathogen dynamics into the model. This allowed the model to predict that, if a pathogen is introduced into the system, it is most likely to persist if it is introduced at a time of year of peak tick questing.

A completely different approach was adopted by Schwarz et al. (2009) who used statistical methods to identify the relationship between vegetation and tick distribution. $I$. ricinus tick count data were correlated with plant communities, and the resulting relationship used to predict I. ricinus distribution across the German nature reserve Siebengebirge, using Geographic Information Systems (GIS). A similar process was undertaken by Braga et al. (2012) to identify the associations between habitat, host densities, temperature and other climatic factors on observed tick abundance at sites across Scotland. The resulting output was used to predict tick abundance over all of Scotland according to GIS-based environmental data, and visualised as a series of raster maps showing predicted tick abundance. The key parameters in this basic algorithm were then altered in accordance with environmental change projections (climate change and woodland expansion), to produce predictions of future tick abundance over Scotland due to environmental change scenarios. 
Jore et al. (2011) also used a statistical method to investigate I. ricinus tick dynamics. A

152 principle component analysis provided a model which explained $67 \%$ of the variation in past

I. ricinus densities in Norway. The study suggests that $I$. ricinus have expanded northwards

154 since 1983.

\subsection{Summary:}

For almost 35 years mathematical models of tick dynamics have been developed. The models have largely focussed on the impact of environmental factors on these dynamics. Field observations show that tick life stages emerge at different points in the season and peak at different times in different geographical regions. In some areas we can have bimodal tick dynamics within a year (e.g. Tagliapietra et al 2011) and in other areas there is only one peak. The models described above have been able to replicate the observed tick dynamics for particular geographical areas, tick species and environmental conditions. However it is clear that in order to be able to predict tick dynamics we would need to have key pieces of information about the environment (and particularly the temperature) in which they live.

Lorenz et al (2014) explicitly looked at the extrapolation of landscape model results to other spatial or temporal systems for Lyme disease and I. scapularis and concluded that models based on measures of vegetation, habitat patch characteristics and herbaceous landcover emerged as effective predictors of observed disease and vector distribution. These would therefore be important characteristics of an area to measure in order to predict these distributions. 
175

176

177

Modelling of tick-borne pathogens has focussed on a small number of pathogens which are important for human or animal health and welfare. The three main systems which have been modelled extensively are louping-ill virus (LIV), western tick-borne encephalitis virus (TBEV) and Borrelia burgdorferi sensu lato, the causative agent of Lyme disease. This section will focus largely on LIV since this pathogen has the largest body of modelling work and it is the area of expertise of the authors. It also illustrates many of the biological features which need to be incorporated into models and so is a good case study for models of other system.

In general transmission of these pathogens can occur in three ways (although also see Park et al. 2001 discussed below for Louping Ill Virus). The most common form of transmission occurs when susceptible ticks feed on infected hosts with virus in their bloodstream (viraemic hosts) and pick up the virus. These ticks then moult into their next developmental stage and when they take their next blood meal then they can pass the pathogen onto a susceptible host, this will be a different individual and can also be a different host species (Labuda and Nuttall 2004). The second method is vertical transmission, for some pathogens infection is passed from adult ticks to eggs and onto larvae (Labuda and Nuttall 2004). Finally, for some hosts and some pathogens there can be non-viraemic or co-feeding transmission in which susceptible ticks feeding near to infectious ticks can pick up infection without the host having a viraemic response (Jones et al 1987).

\subsection{Louping Ill Virus}

A large body of increasingly complex models have been used to help us understand LIV, which is the western-most variant of Western tick-borne encephalitis virus. LIV is 
transmitted by I. ricinus and causes disease in livestock, especially sheep Ovus aries, as well as red grouse Lagopus lagopus scoticus, a valuable game bird. A vaccine has been developed for livestock but not for red grouse that are highly susceptible to the disease, with $78 \%$ mortality rates in experimentally infected birds in the laboratory (Reid 1976). The hosts and transmission cycle of this complex virus system has been recently reviewed (Gilbert 2015), but mathematical models can be extremely useful in helping to identify gaps in our biological knowledge of the system, identifying the relative importance of different host species hosts, and predicting the effectiveness of potential control strategies.

The first mathematical model of LIV was presented by Hudson et al. (1995), where a series of coupled ordinary differential equations describing LIV on red grouse moorland was presented. This model explored the interactions between ticks and red grouse and their role in the dynamics of LIV. The model predicted that grouse alone cannot support a tick population since very few adult ticks feed on grouse, therefore other hosts are required to complete the tick life cycle. Within this model the alternative hosts were mountain hares Lepus timidus, although similar later studies examined the role of red deer Cervus elaphus (Gilbert et al. 2001; Norman et al. 2004) and sheep (Porter et al. 2011). Hudson et al. (1995) also calculated a formula for the conditions for persistence of both ticks and LIV. For tick persistence a sufficient number of hosts (or combination of host types) which can feed all stages of ticks population, with a sufficient number of these being pathogen-transmitting hosts. This threshold formula comes from the basic reproductive rate or number, $\mathrm{R}_{0}$, when $\mathrm{R}_{0}>1$ then the pathogen persists and when $\mathrm{R}_{0}<1$ the pathogen dies out. Some more complex later LIV models have also predicted an eventual 'dilution effect' where pathogen prevalence declines 
222 if there are too many non-pathogen transmitting tick hosts (hosts which do not transmit the

223

224

225

227

228

229

230 pathogen such as deer) compared to competent transmission hosts which causes potential pathogen transmitting bites to be "wasted" and the effect of the pathogen to be diluted (Norman et al. 1999; Gilbert et al. 2001).

Sheep are known to produce a LIV viraemia after infection, and are known to be competent transmission hosts. However, the role of lambs is less well understood; if ewes have been bitten by infected ticks, their young lambs acquire immunity from the virus from drinking the colostrum from their mothers in the first few days or weeks of life. However, as the lambs age this immunity wanes, leaving them at risk of contracting LIV. Thus, lambs could potentially have a role as a reservoir host. Therefore, another differential equation model was created to understand the role that lambs may play as a reservoir of LIV. The model predicted that, whilst in theory large numbers of lambs could act as a reservoir for the virus, it is more likely that, in most situations, these numbers are probably small (Laurenson et al. 2000).

Laurenson et al. (2003) examined the impact of near-eradication of mountain hares on tick burdens and LIV seroprevalence in red grouse, using both empirical data and differential equation models. The models compared the scenario where mountain hares simply act as tick amplifying hosts to a scenario where hares were both tick hosts and non-viraemic transmission hosts. It was found that the model which included non-viraemic transmission produced predictions that fitted the data better than the simpler model did. Laboratory experiments had already identified mountain hares as competent transmission hosts (through supporting non-viraemic transmission between co-feeding ticks) in the laboratory (Nuttall and Jones 1991; Jones et al. 1997). In addition, models have shown that non-viraemic transmission via co-feeding may allow the virus to persist more readily than it would otherwise have done, and allow the virus to persist even in the absence of viraemic hosts if 
the level of non-viraemic transmission is high enough (Norman et al. 2004). However, the

247 Laurenson et al. (2003) study was important in demonstrating that mountain hares can be LIV reservoir hosts in the field. There were large management repercussions to this research, as many grouse moor managers over Scotland began large-scale culls of mountain hares, leading to political issues (reviewed by Harrison et al. 2010; Gilbert 2015). Models again had political impact by providing evidence against culling mountain hares: while the Laurenson et al. (2003) system included only red grouse and mountain hares, most areas in Scotland managed for grouse hunting also have deer. Therefore, Gilbert et al. (2001) modelled a threehost system, including deer as well as red grouse and mountain hares. Importantly, this threehost model predicted that LIV would always persist in the presence of even low densities of deer, even if all mountain hares were culled. This was because red grouse are transmission hosts for the virus while deer, although not competent transmission hosts, are important hosts for all stages of tick, so together both virus and tick life cycles can be maintained. This Gilbert et al. (2001) model has been crucial in the arguments against large-scale mountain hare culls (Harrison et al. 2010; Gilbert 2015).

Mathematical models have also been used in helping identify which pathogen control methods could be theoretically most effective in LIV control. Porter et al. (2011) developed models to predict the effectiveness of using acaricide-treated sheep as a tool to control ticks and LIV in red grouse. The model predicted that the presence of deer limits the effectiveness of such a strategy, but for certain conditions the use of acaricide on sheep could theoretically be a viable method for controlling ticks and LIV providing that high numbers of sheep are treated and acaricide efficacy remains high, while deer densities must be very low (Porter et al. 2011). Due to this predicted adverse impact of deer on the success of treating sheep to control ticks and LIV, and because deer are known to maintain high tick population densities in Scotland and move ticks between habitats (Ruiz-Fons and Gilbert 2010; Jones et al. 2011; 
271 Gilbert et al. 2012), models were then developed to test the theoretical effectiveness of 272 acaricide-treated deer on controlling ticks and LIV (Porter et al. 2013a). The model predicted that treating deer could control ticks and LIV if high acaricide efficacies were maintained and if a large proportion of the deer population was treated. Furthermore, effectiveness was improved if there were only low densities of deer. However, although the model predicted that this control method is theoretically plausible, it is unlikely that the conditions could be met in practical terms, in wild deer. Therefore, using an age-structured differential equation model, including splitting the grouse life cycle to represent the different behaviour between chicks and adults, Porter et al. (2013b) investigated whether acaricide treatment of the grouse themselves could help reduce ticks in the environment and LIV in the grouse population. Again, this was theoretically possible, but in the presence of deer, high acaricide efficacies were required and high proportions of the grouse population treated, were needed for successful control. This is due to the deer amplifying the tick population. These types of models can therefore be of use in decision-making by land managers for choosing disease control options, such as whether to try a certain control method or not depending on the situation in a specific area, taking into account any practical difficulties.

It is generally assumed that LIV is transmitted through ticks biting their hosts, and model parameterisation generally reflects this assumption. However, red grouse chicks frequently eat invertebrates, including ticks (Park et al. 2001). This is a potentially important route of transmission: it has been suggested that $73-98 \%$ of LIV infection in red grouse in their first year could stem from ingestion (Gilbert et al. 2004). Introducing this infection route to LIV modelling has an interesting effect: when using the standard method for calculating the basic reproduction number for the persistence of LIV, then the algebraic results and numerical simulations do not match. The standard method of analysis causes virus persistence to be underestimated, as the ingestion of infected ticks causes a feedback loop where the virus can 
persist with seemingly insufficient hosts (Porter et al. 2011). This phenomenon requires

297 further investigation, as it may indicate interesting gaps in our knowledge of the biology of the LIV system as well as an anomaly in the current modelling approach.

In the LIV models described above there has been no explicit spatial component to the models. However, Watts et al. (2009) investigated the interaction between neighbouring areas by expanding the previously-existing LIV models into a two-patch system with host movement between patches. Comparison with empirical data showed that whilst the onepatch model was a reasonable indicator for tick numbers, it tended to underestimate the prevalence of the LIV. When considering the two-patch model, the results depended largely on finding the appropriate balance of deer movement between the two sites (Watts et al. 2009). Jones et al. (2011) developed a different type of differential equation model, which explicitly tracked the number of ticks on each host, to predict how deer moving ticks from forest onto moorland might affect ticks and LIV in red grouse on the moorland. The assumption was that ticks are more abundant in forest than on moorland, which is supported by empirical data (Ruiz-Fons and Gilbert 2010). This model predicted the highest levels of LIV in moorland to occur where it is bordering forest regions, due to higher tick numbers there. Furthermore, this model was important in examining for the first time the impact of landscape heterogeneity on predicted pathogen levels: virus prevalence was predicted to be higher in landscapes that have larger forest patches, and higher landscape fragmentation, which increases the number of borders between the two habitats (Jones et al. 2011).

\subsubsection{Summary:}

The transmission, persistence and dynamics of Louping Ill virus are complex with many interacting factors to take into account. The focus of the modelling work described above has been on trying to understand the roles that different hosts play in maintaining these 

dynamics. Hosts can play three possible roles, they can either simply act as tick amplifiers (e.g. deer) or they can both amplify ticks and transmit virus (e.g. sheep for viraemic transmission or hares for non-viraemic transmission) or finally they can transmit the

\subsection{Other tick-borne pathogens.}

3.2.1 Tick-borne encephalitis: Tick-borne encephalitis is a neurological disease which is of act as the competent host for the virus.

There are two significant ways in which deer can influence TBEV dynamics. Firstly, as deer are the main host which I. ricinus adults feed on, their presence, as with LIV, has an amplification effect on tick abundance. Secondly, as deer do not support TBEV transmission, very high deer densities can eventually lead to the dilution effect lowering TBEV levels (again similar to model predictions of LIV).

In both 2003 and 2007 Rosa and co-authors extended the models of Norman et. al. (1999) to explicitly include the questing and feeding tick stages and the aggregation of ticks on the hosts. They investigated changes in host densities and different infection pathways to 
determine when the dilution effect might occur. They found the new result that the dilution effect might occur at high densities of disease competent hosts. The authors state that better information on tick demography would be needed before it would be possible to predict whether this effect would happen in the field. However, there is some evidence that this is the case in the TBE system (Perkins 2003).

In 2012 the same Italian group published a pair of papers taking both an empirical and theoretical approach to understanding the effect of deer density of tick distributions on rodents and therefore the risk of TBE. Cagnacci et al. (2012) empirically found a humpshaped relationship between deer density and ticks feeding on rodents, and a negative relationship between deer density and TBE occurrence. Twinned with this, a model was developed by Bolzoni et al. (2012) to explain these findings. They found hump-shaped relationships between deer density and both the number of ticks feeding on rodents and TBEV prevalence in ticks. For low deer densities this can be explained by the tick amplification effect, for high deer densities the virus dilution mechanism dominates the dynamics.

The role of climate change on tick-borne pathogen prevalence was scrutinised by Randolph (2008). In this study, TBEV was used as a case example. A statistical model was used to show that climate change is not enough to explain historical changes in TBE incidence within Europe. An alternative model was presented, showing how the introduction of further factors allowed for a better model fit of the data. Crucially, such a model included socio-economic factors such as unemployment, agricultural practices and income. Zeman et al. (2010) used GIS analysis to similarly find that heterogeneity in TBE trends cannot be fully explained by geographic and climatic factors. However, they also found that the inclusion of socioeconomic conditions could not satisfactorily explain the anomalies. 
368
As with Louping ill the persistence and dynamics of TBE are dependent on host densities and deer play a crucial role in this. Some of the papers described above, particularly the 2003 and 2007 Rosa et al papers present general results which could apply to a number of different tick borne pathogens and, in particular the results that dilution effects are very dependent on tick demography and density dependent constraints are true more generally than just for TBE. In most of the models presented here TBE has been a case study of a model which addresses more general questions.

3.2.2 Lyme Disease: Borrelia burgdorferi s.l. is the suite of spirochete bacteria which causes Lyme disease. This is a pathogen which has a wildlife reservoir but infects humans in the northern hemisphere.

Porco (1999) used a time-independent differential equation model to investigate how the prevalence of B. burgdorferi s.l. in I. scapularis (Say) nymphs is affected by various model parameters. The infectivity of white-footed mice Peromyscus leucopus (a key transmission host in the eastern USA) was predicted to be the parameter which increased $B$. burgdorferi s.l. prevalence the most, whilst a ten-fold increase in the density of deer (which do not transmit the pathogen) significantly reduced B. burgdorferi s.l. prevalence, suggesting that this is another system where the dilution effect can occur.

Zhang and Zhao (2013) presented a seasonal reaction-diffusion model of Lyme disease, utilising it to study the dynamics of the system in bounded and unbounded spaces. For bounded habitats a threshold for pathogen persistence was predicted, whilst for unbounded habitats they were able to predict the speed of pathogen spread. 
In their 2007 paper Ogden et al considered the work of Wilson and Spielman (1985) and hypothesized that the transmission cycles of B. burgdorferi are very efficient in north-eastern North America because the seasonal activity of nymphal and larval I. scapularis is asynchronous. They then developed a simulation model which integrated transmission patterns imposed by seasonal asynchronous nymph and larvae with a model of infection in white footed mice. They parameterised the model for B. burgdorferi and Anaplasma phagocytophilum as examples. They found that duration of host infectivity, transmission efficiency to ticks and co-feeding transmission are the major factors determining fitness of pathogens in I. scapularis in North America.

The same group then wrote a series of papers looking I. Scapularis in Canada where is is established in some places and emerging in others. In Wu et al (2013) they developed a temperature driven map of the basic reproductive number for the ticks and found that for while the geographical extent of suitable tick habitat is expected to increase with climate warming the rate of invasion will also increase. In a subsequent paper Ogden et al (2013a) investigated the speed of $B$. burgdorferi invasion after establishment of ticks. The model showed that the number of immigrating ticks was a key determinant of pathogen invasion and so the authors hypothesized that a 5 year gap would occur between tick and $B$. burgdorferi invasion in Eastern Canada but a much shorter gap in Central Canada. This was consistent with empirical evidence. This was investigated more generally in Ogden et al (2013b).

\subsubsection{Summary}

Borrelia burgdorferi is another pathogen for which the dilution effect appears to occur. In this case rodents are the main reservoir host and B. burgdorferi is emerging in a number of different areas as the tick hosts expand their range in response to climate change or socioeconomic factors. 
414 More generally Hartemink et al. (2008) determined ways of characterising the basic 415 reproductive number in a tick-borne pathogen system which has multiple transmission routes 416 using the next generation matrix (e.g. Diekmann et al. 2010). They showed that the 417 complexities of the tick transmission cycle can be overcome by separating the host population into epidemiologically different types of individuals and constructing a matrix of reproduction numbers. They then used field and experimental data to parameterise this nextgeneration matrix for B. burgdorferi s.l. and TBEV.

421 Dunn et al (2013) used a general model of tick-borne pathogens to study the basic reproductive number and found that the transmission efficiency to the ticks, the survival rate from feeding larvae to feeding nymphs and the fraction of nypmhs to find a competent host are the most important factors in determining $\mathrm{R}_{0}$.

425 Another general tick-borne pathogen model was created by Zeman (1997), where reported 426 cases of disease were smoothed over to create risk maps for Lyme disease and TBE in 427 Central Bavaria. This study indicated that B. burgdorferi s.l. is wider spread than TBEV, but that both pathogens share the same main foci. Similarly, Hönig et al. (2011) assessed the suitability of various habitats for supporting I. ricinus ticks, creating a model with which they were able to create a tick-borne pathogen risk map for South Bohemia, which was compared to clinical cases of TBE for validation. The model suggested that the areas most suitable for tick-borne pathogens were along river valleys. However, when human activity is taken into account, the surroundings of large settlements are equally likely to provide tick-borne pathogen cases. 
435 Another aspect of transmission which is considerably less well understood is the pattern of 436 aggregation of ticks on hosts. Ferreri et al (2014) analysed a nine year time series of Ixodes

437 ricinus feeding on Apodemus flavicollis mice, the reservoir host for TBE in Trentino, 438 Northern Italy. The tail of the distribution of the number of ticks per host was fitter to three 439 theoretical distributions. The impact of these distributions on pathogen transmission was 440 investigated using a stochastic model. Model simulations showed that there were different outcomes of disease spread with different distribution laws amongst ticks.

442 The models discussed above are not an exhaustive list, but do describe models which help us 443 to understand many of the different complexities of tick-borne pathogen systems, and 444 showcase the diversity of models now being developed for a wide range of end uses.

\section{KNOWLEDGE GAPS AND FUTURE DIRECTIONS}

447 As we have seen mathematical models have been used for more than 30 years to help to 448 predict tick dynamics and subsequently pathogen dynamics. The models presented here have 449 been used in two ways, firstly to predict when tick densities are at their peak within a year and how that peak varies with environmental factors. Secondly, they have been used to predict pathogen persistence for different combinations of available host species with different transmission competencies. In particular, they have looked at the interaction between tick amplifying hosts and disease transmitting hosts and how densities of these hosts could be manipulated to control the disease.

One of the problems of these modelling studies is the difficulty in gathering empirical data to validate the model results. This is largely because there is a great deal of variability between sites in terms of habitat cover, microclimate and host densities. This is not unique to the tick 
system, it is difficult for a number of reasons to carry out experiments in natural systems. It is also difficult to measure realistic tick densities (e.g. Dobson 2014).

However, most of the models described here have succeeded in doing some type of validation and they provide useful qualitative results.

Future modelling approaches are likely to be focussed in three areas. One is to look at spatial patterns of tick and disease risk, and in particular to link environmental information in GIS systems to models of tick and pathogen dynamics in a mechanistic way. These models can then be used to predict the impact of climate change on tick and disease risk across a given geographical region. This type of modelling is currently being carried out at the University of

Stirling for Scotland. The advantage of this type of modelling is that it is generalizable and could be applied to any country with the right type of environmental data available in GIS form. It can also predict risks are going to change over time rather than only looking at the end points as has been done before (e.g. Braga et al 2012).

If we can identify which areas are going to have significant increases in disease risk then we can inform policy makers and target control efforts. For example, if we could identify which areas are going to have higher and lower Lyme disease risk then we could target efforts to educate the public on how to avoid being bitten in those high risk areas.

The second direction which we predict tick modelling will move is to further a new modelling technique which was introduced in Jones et al (2011). In that paper the authors developed a model which keeps track of the number of hosts with a particular number of ticks on it 
481 Financial support:

482 AJW is supported by a Partnership Impact PhD Studentship funded by both the University of 483 Stirling and the James Hutton Institute. 
484

485

486

487

488

489

490

491

492

493

494

495

496

497

498

499

500

501

502

503

504

Bolzoni, L., Rosa, R., Cagnacci, F., Rizzoli, A. (2012). Effect of deer density on tick infestation of rodents and the hazard of tick-borne encephalitis. II: Population and infection models. International Journal for Parasitology 42, 373-381.

Braga, J.F. (2012) Predicting current and future tick abundance across Scotland. Masters Thesis, University of Aberdeen, UK.

Cagnacci, F., Bolzoni, L., Rosa, R., Carpi, G., Hauffe, H. C., Valent, M., Tagliapietra, V., Kazimirova, M., Koci, J., Stanko, M., Lukan, M., Henttonen, H., Rizzoli, A. (2012). Effects of deer density on tick infestation of rodents and the hazard of tick-borne encephalitis. I: Empirical assessment. International Journal of Parasitology 42, 365-372.

Cooksey, L. M., Haile, D. G., Mount, G. A. (1990). Computer simulation of Rocky Mountain Spotted Fever transmission by the Amercian Dog tick (acari, Ixodidae). Journal of Medical Entomology 27(4), 686-696.

Diekmann, O., Heesterbeek, J. A. P., Roberts, M. G. (2010). The construction of nextgeneration matrices for compartmental epidemic models. Journal of the Royal Society Interface 7(47), 873-885.

Dobson, A., Finnie, T., Randolph, S. (2011a). A modified matrix model to describe the seasonal population ecology of the European tick Ixodes ricinus. Journal of Applied Ecology 48(4), 1017-1028. doi: 10.1111/j.1365-2664.2011.02003.x.

Dobson, A., Randolph, S. (2011b). Modelling the effects of recent changes in climate, host density and acaricide treatments on population dynamics of Ixodes ricinus in the UK. Journal of Applied Ecology 48(4), 1029-1037. doi: 10.1111/j.1365-2664.2011.02004.x. 
505

506

507

508

509

510

511

512

513

514

515

516

517

518

519

520

521

522

523

524

525

526

Dobson, A. (2014) History and complexity in tick-host dynamics: discrepancies between 'real' and 'visible' tick populations. Parasites and Vectors 7: 231

Dunn, J.M., Davis, S., Staecy, A, Diuk-Wasser, M.A. (2013) A simple model for the establishment of tick-borne pathogens of Ixodes scapularis; A global sensitivity analysis of $\mathrm{R}_{0}$. Journal of Theoretical Biology. 335, 213-221.

Ferreri, L. Giacobini, M., bajardi, P., Bertolotti, L., Bolzoni, L., Tagliapietre, V., Rizzoli, A., Rosa, R. (2014) Pattern of tick aggregation on mice: larger than expected distribution tail enhances the spread of tick-borne pathogens. PLOS computational biology. 10(11) e1003931.

Gardiner, W. P. Gettinby, G. and Gray, J. S. (1981). Models based on weather for the development phases of the sheep tick, Ixodes ricinus L. Veterinary Parasitology 9, 75-86.

Gilbert, L. (2015) Louping ill virus in the UK: a review of the hosts, transmission and ecological consequences of control. Experimental and Applied Acarology. DOI 10.1007/s10493-015-9952-x pp 1-12 First online: 24 July 2015

Gilbert, L., Aungier, J. and Tomkins, J. L. (2014). Climate of origin affects tick (Ixodes ricinus) host-seeking behaviour in response to temperature: implications for resilience to climate change? Ecology and Evolution 4 (7), 1186-1198. doi: 10.1002/ece3.1014.

Gilbert L., Norman R., Laurenson K. M., Reid H. W., Hudson P. J. (2001). Disease persistence and apparent competition in a three-host community: an empirical and analytical study of large-scale, wild populations. Journal of Animal Ecology 70(6), 1053-1061. 

(2004) Ticks need not bite their red grouse hosts to infect them with louping ill virus.

530 Gilbert, L., Maffey, G., Ramsay, S. L., Hester, A. J. (2012). The effect of deer 531 Management on the abundance of Ixodes ricinus in Scotland. Ecological Applications 22(2), 532 658-667.

533

Gray, J. S. (1987) Mating and behavioural diapause in Ixodes ricinus L. Experimental and 534 applied acarology $3,61-71$.

535

536 Gray, J. S. (1998) The ecology of ticks transmitting Lyme borreliosis. Experimental and 537 applied acarology. 22(5) 249-258.

Hancock P., Brackley R., Palmer S. (2011). Modelling the effect of temperature variation 539 on the seasonal dynamics of Ixodes ricinus tick populations. International Journal for $540 \quad$ Parasitology 41(5), 513-522.

541 Harrison, A., Newey, S., Gilbert, L., Haydon, D. T., Thirgood, S. (2010). Culling wildlife 542 hosts to control disease: mountain hares, red grouse and louping ill virus. Journal of Applied 543 Ecology 47(4), 926-930.

544 Hartemink, N. A., Randolph, S. E., Davis, S. A., Heesterbeek, J. A. P. (2008). The basic 545 reproduction number for complex disease systems: Defining R-0 for tick-borne infections. American Naturalist 171(6), 743-754. 
547 Hönig V., Švec, P., Masař, O., Grubhoffer, L. (2011). Tick-borne disease risk model for 548 South Bohemia (Czech Republic). In GIS Ostrava 2011, Eight International Symposium, 549 Proceedings“. ISBN 978-80-248-2406-2. 255-268 p.

550 Hudson, P. J., Norman, R., Laurenson, M. K., Newborn, D., Gaunt, M., Gould, E., Reid, 551 H., Bowers, R. G., and Dobson, A. P. (1995). Persistence and Transmission of Tick-borne 552 Viruses: ixodes ricinus and Louping Ill virus in Red Grouse populations. Parasitology 111, 553 s49-s58.

Jones, L.D., Davies C.R., Steele, C.M and Nuttall, P.A., (1987) A novel mode of arbovirus transmission involving a nonviraemic host. Science 237, 775-7.

Jones, L. D., Gaunt, M., Hails, R. S., Laurenson, K., Hudson, P. J., Reid, H., Henbest,P., ticks co-feeding on mountain hares (Lepus timidus). Medical and veterinary Entomology 11, 172-176.

Jones, E. O., Webb, S. D., Ruiz-Fons, F. J., Albon, S., Gilbert, L. (2011). The effect of 561 landscape heterogeneity and host movement on a tick-borne pathogen. Theoretical Ecology 4(4), 435-448.

Jore, S., Viljugrein, H., Hofshagen, M., Brun-Hansen, H., Kristoffersen, A. B., Nygård, 564 K., Brun, E., Ottesen, P., Sævik, B. K., Ytrehus, B. (2011). Multi-source analysis reveals latitudinal and altitudinal shifts in range of Ixodes ricinus at its northern distribution limit. Parasites \& Vectors 4, Article Number 84. 
Laurenson, M. K., Norman, R., Reid, H. W., Pow, I., Newborn, D., Hudson, P. J. (2000). The role of lambs in louping-ill virus amplification. Parasitology 120, 97-104.

Laurenson, M. K., Norman, R. A., Gilbert, L., Reid, H. W. and Hudson, P. J. (2003). Identifying disease reservoirs in complex systems: mountain hares as reservoirs of ticks and louping-ill virus, pathogens of red grouse. Journal of Animal Ecology 72, 177-185.

Lorenz, A., Dhingra, R., Chang, H.H., Bisanzio, D., Liu, Y and Remais, J.V. (2014) Inter-model comparison of the landscape determinants of vector-borne disease: Impliocations for epidemiological and entomological risk modeling. PLOS one 9(7) e103163

Medlock, J., Hansford K. M., Bormane A., Derdakova M., Estrada-Peña A., George J. C., Golovljova I., Jaenson T. G., Jensen J. K., Jensen P. M., Kazimirova M., Oteo J. A., Papa A., Pfister K., Plantard O., Randolph S. E., Rizzoli A., Santos-Silva M. M., Sprong H., Vial L., Hendrickx G., Zeller H., Van Bortel W. (2013). Driving forces for changes in geographical distribution of Ixodes ricinus ticks in Europe. Parasites \& Vectors. 6:1. doi: 10.1186/1756-3305-6-1.

Mount, G. A., Haile, D. G. (1989). Computer simulation of population dynamics of the American dog tick (Acari: Ixodidae). Journal of Medical Entomology 26(1), 60-76.

Norman, R., Bowers, R. G., Begon, M. and Hudson, P. J. (1999). Persistence and Dynamics of Louping Ill in Relation to Host Abundance. Journal of Theoretical Biology 200, $111-118$.

Norman, R., Ross, D., Laurenson, M. K., and Hudson, P. J. (2004). The role of nonviraemic transmission on the persistence and dynamics of a tick-borne virus Louping ill in 
590

591

592

593

594

595

596

597

598

599

600

601

602

603

604

605

606

607

608

609

610

611

Red Grouse (Lagopus lagopus scoticus) and Mountain Hares (Lepus timidus). Journal of Mathematical Biology 48, 119-134.

Nuttall, P. A. and Jones, L. D. (1991). Non-viraemic tick-borne virus transmission: mechanism and significance. Book Author(s): Dusbabek, Frantisek; Buvka, Vladimir. Modern acarology. Volume II: proceedings of the 8 International Congress of Acarology held in Ceske Budejovice, Czechoslovakia, 6-11 August 1990. Pages 3-6.

Ogden, N.H., Lindsay, A.R., Charron, D., Beauchamp, G., Maarouf, A, O’Callaghan, C.J., Waltner-Tiews, D., Barker, I.K. (2004) Investigation of the relationships between temperature and development rates of the tick Ixodes scapularis (Acari: Ixodidae) in the laboratory and field. Journal of Medical Entomology, 41, 622-633.

Ogden, N.H., Bigras-Poulin, M., O’Callaghan, C.J., Barker, I.K., Lindsay, L.R., Maarouf, A., Smoyer-omic, K.E., Waltner-Toews, D. and Charron, D. (2005) A dynamic population model to investigate effects of climate on geographic range and seasonality of the tick Ixodes scapularis. International Journal for Parasitology 35 375-389.

Ogden, N.H., Bigras-Poulin, M., O’Callaghan, C.J., Barker, I.K., Kurtenbach, K., Lindsay, L. R. and Charron, D.F. (2007) Vector seasonality, host infection dynamics and fitness of pathogens transmitted by the tick Ixodes scapularis. Parasitology 134 209-227.

Ogden, N.H., Lindsay, L.R. and Leighton, P.A. (2013a) Predicting the rate of invasion of the agent of Lyme disease Borrelia burgdorferi. Journal of Applied Ecology. 50 510-518.

Ogden, N.H., Mechai, S and Margos, G. (2013b) Changing geographic ranges of ticks and tick-borne pathogens: drivers, mechanisms and consequences for pathogen diversity. Frontiers in cellular and infection microbiology. 3 article 46. 
Hudson, P., J. (2001). The role of invertebrates in the diet, growth and survival of red grouse

Perkins, S. (2003) Transmission dynamics of tick-borne diseases associated with small mammals. PhD thesis, University of Stirling, Scotland, UK.

617 Perret, J. L., Guigoz, E., Rais, O., Gern, L. (2000). Influence of saturation deficit and temperature on Ixodes ricinus tick questing activity in a Lyme borreliosis-endemic area (Switzerland). Parasitology Research 86(7), 554-557.

Porco, T. C. (1999). A mathematical model of the ecology of Lyme disease. IMA Journal of Mathematics Applied in Medicine and Biology 16, 261-296.

Porter, R., Norman, R., Gilbert, L. (2011). Controlling tick-borne diseases through domestic animal management: a theoretical approach. Theoretical Ecology 4(3), 321-339.

Porter, R. Norman, R.A. and Gilbert, L. (2013a). An alternative to killing? Treating 625 wildlife hosts to protect a valuable species from a shared parasite. Parasitology 140, 247-25.

Porter, R. Norman, R., Gilbert, L. (2013b). An empirical model to test how ticks and 627 louping ill virus can be controlled by treating red grouse with acaricide. Medical Veterinary 628 Entomology 27(3), 237-246. 10.

Randolph, S. E., Rogers, D. J. (1997). A generic population model for the African tick 
631 Randolph S. E., Green, R., Hoodless, A., Peacey, M. F. (2002). An empirical, quantitative 632 framework for the seasonal population dynamics of the tick Ixodes ricinus. International 633 Journal for Parasitology 32(8) 979-989.

634 Randolph, S. E. (2008). Dynamics of tick-borne disease systems: minor role of recent 635 climate change. Revue Scientifique et Technique-Office International des Epizooties 27(2), 636 $367-281$.

637

Reid, H. W (1976). The epidemiology of Louping-ill. In: Tick-borne Diseases and their 638 vectors. (Wilde, J. K. H., ed.).Proc. Int. Conf. held in Edinburgh September 27 October 1, 639 1976.

Rosa, R., Pugliese, A., Norman, R., Hudson, P.J. (2003) Thresholds for disease persistence 641 in models for tick-borne infections including non-viraemic transmission, extended feeding and tick aggregation. Journal of Theoretical Biology. 224(3) 359-376.

Rosa, R. and Pugliese, A. (2007) Effects of tick population dynamics and host densities on 644 the persistence of tick-borne infections. Mathematical Biosciences 208 216-240.

645

Ruiz-Fons, F. and Gilbert, L. (2010). The role of deer (Cervus elaphus and Capreolus 646 capreolus) as vehicles to move ticks Ixodes ricinus between contrasting habitats. 647 International Journal for Parasitology 40(9), 1013-1020. doi: 10.1016/j.ijpara.2010.02.006.

Schwarz, A., Maier, W. A., Kistemann, T., Kampen, H. (2009). Analysis of the 649 distribution of the tick Ixodes ricinus L. (Acari: Ixodidae) in a nature reserve of western Germany using Geographic Information Systems. International Journal of Hygiene and 

H.C. and Rizzoli, A. (2011) Saturation deficit and deer density affect questing activity and local abundance of Ixodes ricinus (Acari, Ixodidae) in Italy. Veterinary Parasitology. 183, $114-124$.

Tomkins, J. L., Aungier, J., Hazel, W., Gilbert, L. (2014). Towards an evolutionary understanding of host seeking behaviour in the Borrelia burgdorferi sensu lato vector Ixodes ricinus: data and theory. PloS ONE 9(10), e110028. doi:10.1371/journal.pone.0110028.

\section{Watts, E. J., Palmer, S. C. F., Bowman, A. S., Irvine, R. J., Smith, A., Travis, J. M. J.} (2009). The effect of host movement on viral transmission dynamics in a vector-borne disease system. Parasitology 136(10), 1221-1234.

Wilson, M.L. and Spielman, A. (1985) Seasonal activity of immature Ixodes dammini (Acari:Ixodidae). Journal of Medical Entomology. 26 408-414

Wu, X., Duvvuri, V.R., Lou, Y., Ogden, N.H., Pelcat, Y. and Wu, J (2013) Developing a temperature-driven map of the basic reproductive number of the emerging tick vector of Lyme disease Ixodes scapularis in Canada. Journal of Theoretical Biology. 319. 50-61.

Zeman, P. (1997). Objective Assessment of Risk Maps of Tick-Born Encephalitis and Lyme Borreliosis Based on Spatial Patterns of Located Cases. International Journal of Epidemiology 26(5), 1121-1130.

Zeman, P., Pazdiora, P., Benes, C. (2010). Spatio-temporal variation of tick-borne encephalitis (TBE) incidence in the Czech Republic: Is the current explanation of the disease's rise satisfactory? Ticks and Tick-borne Diseases 1, 129-140. 
673 Zhang, Y., Zhao, X.-Q. (2013). A Reaction-Diffusion Lyme Disease Model with 674 Seasonality. Society for Industrial and Applied Mathematics 73(6), 2077-2099.

675

676 
677 Figure 1: Schematic diagram of the I. Ricinus lifecycle with the type of host that they are able to feed on at each stage.
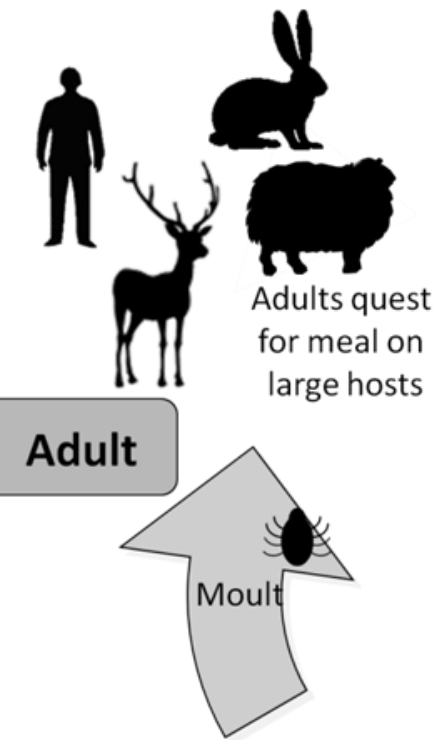

679
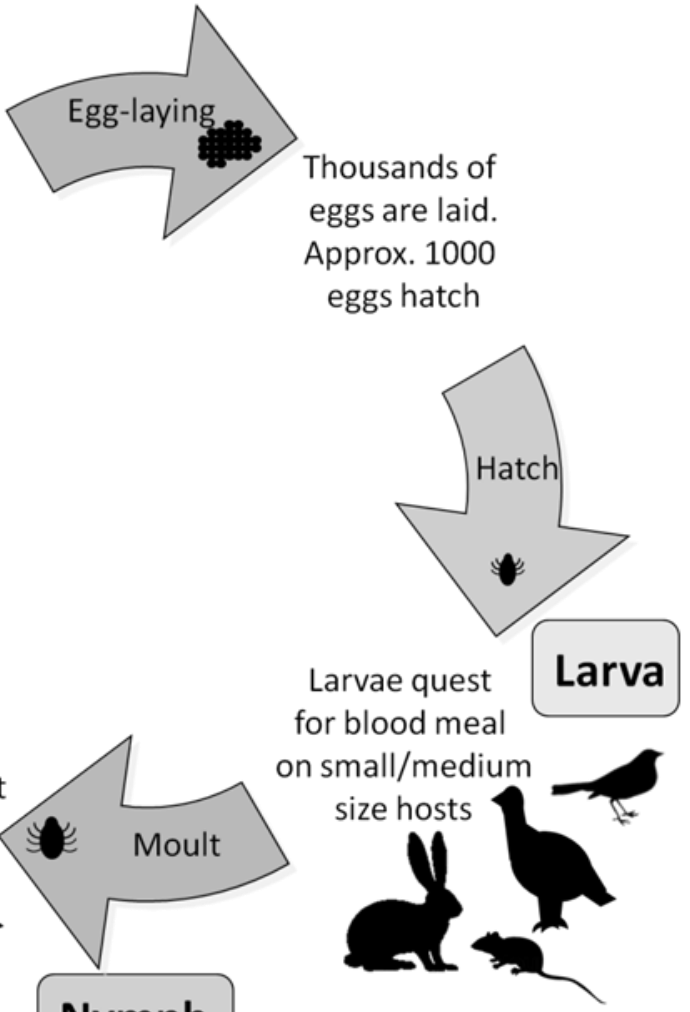